
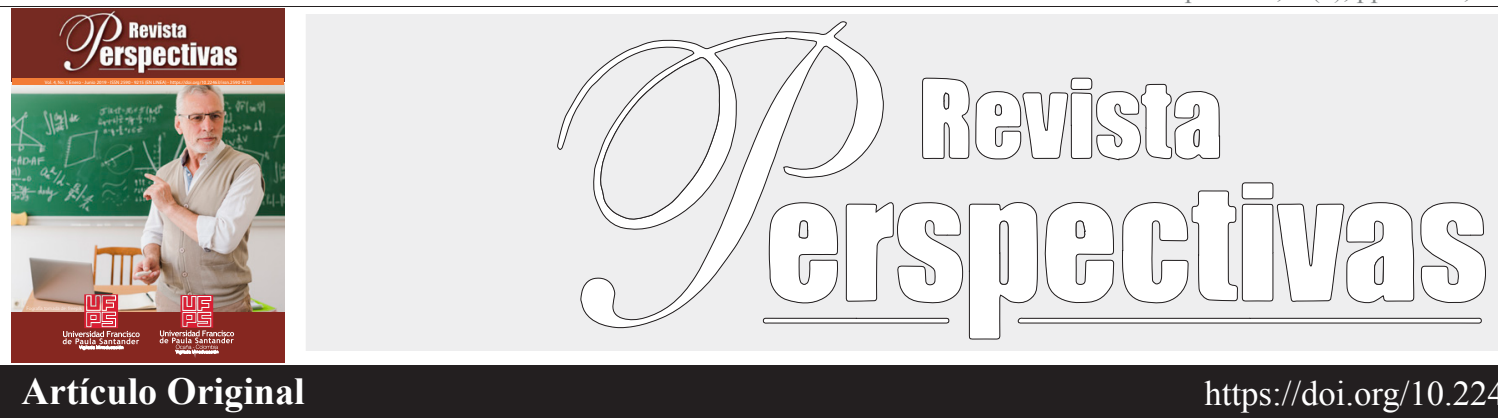

Artículo Original

https://doi.org/10.22463/25909215.1760

\title{
La praxis del profesor de matemáticas: el caso de las competencias transversales en un programa de ingeniería de sistemas
}

The mathematics lecturer's practice: the case of transversal competences in a program of systems engineering

A prática do professor de matemática: o caso das competências transversais no programa de engenharia de sistemas

Elkin Gelvez-Almeida ${ }^{a^{*}}$, Marisela Vivas-García ${ }^{\mathrm{b}}$

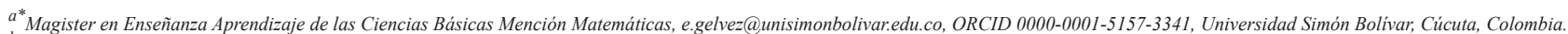

${ }^{b}$ Doctor en Innovaciones Educativas, m.vivas@unisimonbolivaredu.co, ORCID 0000-0002-8941-4562, Universidad Simón Bolivar, Cúcuta, Colombia.

Cómo citar: Gelvez-Almeida, E., \& Vivas-García, M. (2019). La praxis del profesor de matemáticas: el caso de las competencias transversales en un programa de ingeniería de sistemas. Perspectivas, 4(1), 54-59.

Recibido: Agosto 20, 2018; Aceptado: Noviembre 01, 2018

\begin{tabular}{|c|c|}
\hline & RESUMEN \\
\hline Palabras Clave: & $\begin{array}{l}\text { El presente artículo se deriva de la investigación titulada "Competencias transversales en matemáticas que fortalecen la } \\
\text { formación profesional del ingeniero de sistemas de la Universidad Simón Bolívar sede Cúcuta" cuyo objetivo general fue }\end{array}$ \\
\hline Aprendiz & as de los profesores de matemáticas y las técnicas e instrumentos aplicados para la valoración de las \\
\hline Evaluación autentica & ersales. Metodológicamente sigue un enfoque mixto, diseño de campo- transversal y nivel descriptivo. \\
\hline Formación por competencias & Los sujetos que conformaron la muestra fueron 3 profesores y 75 estudiantes de los diferentes cursos de matemáticas del \\
\hline Praxis del profesor & $\begin{array}{l}\text { programa académico de ingeniería de sistemas. Las técnicas empleadas para la recolección de datos y el análisis de los } \\
\text { mismos fueron: la encuesta mediante cuestionario estructurado, y el análisis documental y estadístico utilizando software } \\
\text { especializados como Atlas.ti y el SPSS Statistics respectivamente. Los resultados reflejan la relación existente entre la } \\
\text { percepción de los estudiantes y la praxis pedagógica del profesor de matemáticas. }\end{array}$ \\
\hline
\end{tabular}

ABSTRACT

Keywords:

Meaningful learning Authentic evaluation

Transversal competence

Lecturer practice
This paper is derived from the research entitled "Transversal competences in mathematics that strengthen the professional training of the systems engineer at the Simon Bolivar University Cucuta campus" whose general objective was to analyze the practices of mathematics teachers, and the techniques and instruments applied for the assessment of transversal competences. Methodologically follows a mixed approach, cross-field design, and descriptive level. The subjects that made up the sample were 3 lecturers and 75 students from the different mathematics courses of the systems engineering academic program. The techniques used for data collection and analysis were: the survey using a structured questionnaire, and documentary and statistical analysis using specialized software such as Atlas.ti and SPSS Statistics respectively. The results reflect the relationship between the perception of students and the pedagogical practice of the mathematics lecturer.

Palavras chave:
Aprendizagem significativa
Avaliação autêntica
Formação de habilidades
Prática de professores

\section{RESUMO}

Este artigo é derivado da pesquisa intitulada "habilidades interdisciplinares em matemática que fortalecem a formação profissional do engenheiro de sistemas da Universidade Simon Bolivar sede Cucuta", cujo objetivo geral foi analisar as práticas dos professores de matemática e as técnicas e instrumentos aplicados para a avaliação das habilidades interdisciplinares. Metodologicamente segue uma abordagem mista, design cross-field, e nivel descritivo. Os sujeitos que compuseram a amostra foram 3 professores e 75 estudantes dos diferentes cursos de matemática do programa acadêmico de engenharia de sistemas. As técnicas utilizadas para coletar e analisar os dados foram: a enqueta utilizando um questionário estruturado, e análise documental e estatística utilizando softwares especializados como Atlas.ti e SPSS Statistics respectivamente. Os resultados refletem a relação entre a percepção dos estudantes e a prática pedagógica do professor de matemática.

\section{Introducción}

Uno de los primeros pasos para transformar la educación superior en el siglo XXI es identificar la práctica de los profe- sores, en este caso del área de matemáticas del programa de ingeniería de sistemas de la Universidad Simón Bolívar sede Cúcuta, y así poder diseñar estrategias de mejora de acuerdo a la población objeto de estudio. En este sentido y para iniciar, se toma como referencia los aportes de Ferrer (1997), quien 
afirma que el diseño de cada estructura de formación profesional debe estar conformado por dos partes; las competencias transversales asociadas al sistema productivo del campo de la profesión, y las competencias específicas asociadas a los módulos profesionales.

Por su parte, Tobon (2008) considera que la formación por competencias ha evolucionado al punto de convertirse en una política educativa internacional, teniendo en cuenta especialmente las contribuciones conceptuales y metodológicas de investigadores desde la década de los sesenta, la inclusión como política educativa por muchas naciones, y la marcha de diversos proyectos investigativos en el campo de la educación que tienen como base las competencias (Gelvez, 2017). En este sentido, instituciones gubernamentales como el Instituto Colombiano para el Fomento de la Educación Superior (ICFES) mantiene constantes evaluaciones por competencias en los diferentes niveles de formación por medio de sus pruebas Saber, teniendo en cuenta que el Ministerio de Educación Nacional (MEN, 2008), las considera fundamentales y propone su desarrollo desde la educación básica hasta la superior.

Teniendo en cuenta lo anterior, es importante destacar que esta investigación se centró en el campo de las competencias transversales, y en este sentido se tomaron como antecedentes trabajos alrededor de ello realizados por Benhayón (2006), Urrego-Giraldo y Geraldo (2009), Leal (2014) y Torrado (2015), quienes orientaron el desarrollo conceptual y metodológico de esta investigación, tomando así como base fundamental el concento de competencia (su estructura y el proceso de enseñanza y aprendizaje basado en competencias) y la formación profesional.

Con relación al concepto de competencia, este ha tenido una evolución enorme gracias a los aportes teóricos de varios autores de diferentes áreas, iniciando en la década de los sesenta con la lingüística de Chosky y la psicología conductual de Skinner (Tobon, 2006). Asimismo la psicolingüística plantea desde la base de la interacción de la persona con el entorno, la competencia comunicativa como el empleo efectivo del lenguaje y de la lingüística en situaciones específicas de comunicación (Hymes, 1996), mientras que la psicología cognitiva destaca con la teoría de las inteligencias múltiples de Gardner (1987), y la inteligencia práctica de Sternberg (1997). Por su parte, la psicología laborar también se encuentra presente con los aportes de McClelland (1973), donde las competencias surgen como una forma de determinar las características que un empleado debe poseer para que las empresas alcancen los más altos niveles de productividad y rentabilidad.

Como se puede observar, el concepto de competencia ha recibido aportes de diferentes áreas del conocimiento, especialmente la psicología. Ahora bien, con relación al proceso de enseñanza y aprendizaje, según Díaz y Hernández (1999) las competencias se encuentran involucradas en la promoción de aprendizajes significativos, en concordancia los autores mencionan como estrategias de enseñanza los objetivos o propósitos de aprendizaje que incide en la activación de conocimientos previos, los organizadores previos y analogías para potenciar el enlace entre los conocimientos previos y la información que se ha de aprender, preguntas intercaladas e ilustraciones que inciden en mantener la orientación, y por último los mapas conceptuales y redes semánticas que promueven una organización más adecuada de la información que se ha de aprender.

Los autores mencionados dividen las estrategias de aprendizaje en tres procesos, por un lado se encuentra el aprendizaje memorístico que tiene como propósito la recirculación de la información y el repaso simple, por otro lado se encuentra el recuento, que se refiere a la recuperación y tiene como finalidad la evocación de la información, y finalmente está la elaboración y organización, que pretenden estructurar la información logrando la interpretación significativa en su proceso.

Para la Universidad Simón Bolívar, las competencias se encuentran divididas en competencias básicas transversales y competencias profesionales (ver figura 1). Esta investigación se centró en indagar la praxis del profesor de matemáticas en torno a las competencias básicas transversales, por lo que es importante identificar las dimensiones institucionales.

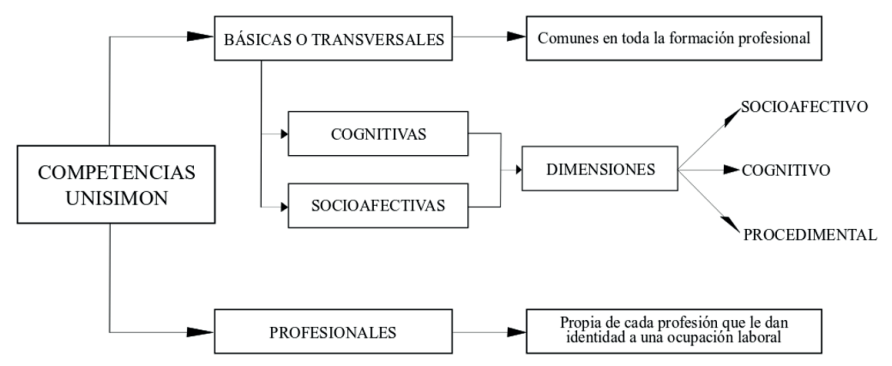

Figura 1. Competencias Universidad Simón Bolívar Fuente: Gelvez (2016)

Finalmente, la formación profesional se inclina hacia la integralidad, apareciendo constantemente en los planes de estudio de los diferentes programas académicos, pero pocas veces puesto en práctica en los ambientes de aprendizaje (Trejos, 2011), situación a indagar en esta investigación.

\section{Materiales y métodos}

Con el propósito de analizar la metodología que implementan los profesores en clase, así como las técnicas e instrumentos que utilizan en la valoración de competencias transversales en las asignaturas de matemáticas de ingeniería de sistemas, se realizó una investigación mediante un enfoque mixto de nivel descriptico, ya que se buscó especificar características, propiedades y rasgos importantes del objeto de estudio (Hernández, Fernández y Baptista, 2014), mientras que el diseño fue de campo-transversal, ya que la información se recolectó directamente de las personas pertenecientes a la muestra una sola vez en un único momento en el tiempo, sin realizar ninguna manipulación deliberada en las variables (Navarro 2009).

En cuanto a la población, estuvo conformada por tres 
profesores y 93 estudiantes de los cursos de álgebra y trigonometría, cálculo diferencial, álgebra lineal, cálculo integral, cálculo de varias variables, y ecuaciones diferenciales del programa académico de Ingeniería de Sistemas de la Universidad Simón Bolívar Sede Cúcuta. Por su parte, para el caso de los estudiantes se trabajó con una muestra probabilística calculada matemáticamente con precisión, seleccionando sus integrantes de forma aleatoria con el fin de que todos tengan la misma probabilidad se ser escogidos (Balbo, 2008), en este sentido se decidió trabajar con un nivel de confianza de $95 \%$, un margen de error de 5\% y una distribución de 50\% como se muestra en la ecuación 1 .

$$
n=\frac{N * Z^{2} * p * q}{d^{2} *(N-1)+Z^{2} * p * q}
$$

\author{
Donde \\ $n=$ Muestra \\ $N=$ Población \\ $Z=$ Nivel de confianza (1.96 para el 95\%) \\ $p=$ distribución \\ $q=1-p$ \\ $d=$ Margen de error
}

En este sentido, la muestra quedó constituida por un total de 75 estudiantes de los diferentes cursos.

Ahora bien, con relación a las técnicas e instrumentos de recolección de información, para el caso de los datos cuantitativos se implementó como técnica la encuesta por medio de un cuestionario estructurado que fue aplicado a los estudiantes pertenecientes a la muestra. Además, el instrumento fue validado por dos expertos temáticos y un metodólogo, garantizando que los ítems midieran de forma adecuada los contenidos y objetivos correspondientes (Navarro 2009), mientras que la confiabilidad estuvo dada por el Alfa de Crombach (Tabla 1) obteniendo un resultado muy favorable.

Tabla 1. Alfa de Crombach

\begin{tabular}{cccc}
\hline Dimensiones & Items & Alfa de Crombach & $\begin{array}{c}\text { Magnitud de } \\
\text { confiabilidad }\end{array}$ \\
\hline Didáctica & $1-17$ & 0,85 & Muy Alta \\
Materiales y recursos & $18-21$ & 0,55 & Moderada \\
Evaluación & $22-33$ & 0,74 & Alta \\
Todas las dimensiones & $1-33$ & 0,89 & Muy Alta \\
\hline
\end{tabular}

Fuente: Gelvez (2016)

Por su parte, para el caso de la información cualitativa, se realizó una revisión, registro y análisis documental de los programas analíticos, registros de listas de asistencia, materiales y otros artefactos tanto institucionales como del profesor.

\section{Resultados y discusiones}

A continuación, se presenta la descripción de los resultados obtenidos en la aplicación de los instrumentos a los integrantes de la muestra, teniendo en cuenta las tres dimensiones investigadas (didáctica, materiales y recursos, y evaluación). Para la interpretación de las gráficas que se presentan a continuación, es importante conocer la escala de valoración implementada en los instrumentos, que se encuentran distribuido de la siguiente forma:

- 5,0 Totalmente de acuerdo

- 4,0 De acuerdo

- 3,0 Ni de acuerdo ni en desacuerdo

- 2,0 en desacuerdo

- 1,0 Totalmente en desacuerdo

Ahora bien, teniendo en cuenta la escala de valoración, se presenta de forma general en la figura 2, la percepción de los estudiantes frente a la dimensión de didáctica por medio de un diagrama de caja y bigotes. Esta dimensión busca indagar en los estudiantes de Ingeniería de Sistemas sobre las estrategias de enseñanza aplicada por los profesores de matemáticas, así como las estrategias de aprendizaje implementada por ellos.

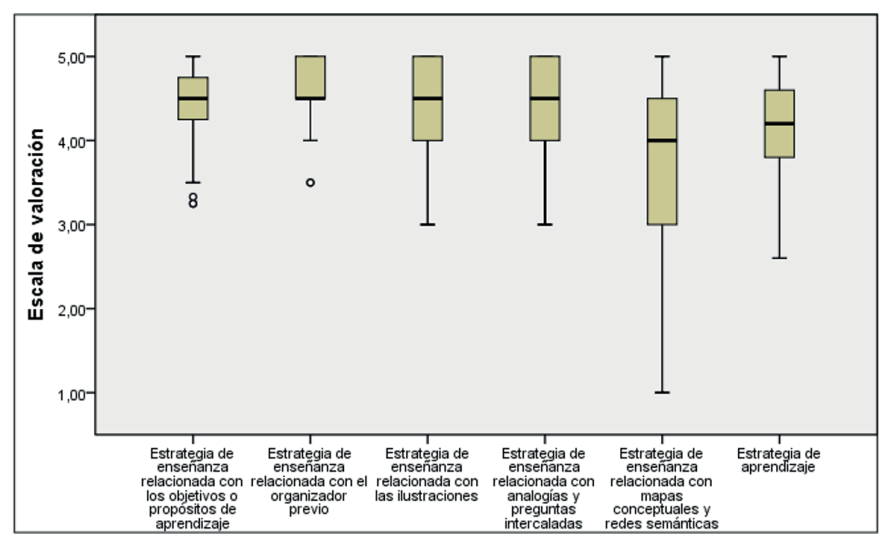

Figura 2. Percepción de los estudiantes frente a las estrategias de enseñanza y aprendizaje

En relación con las estrategias de enseñanza relacionadas con los objetivos o propósitos de aprendizaje, organizadores previos, ilustraciones, y analogías y preguntas intercaladas, se evidencia una buena percepción por parte de los estudiantes, teniendo en cuenta que la mayor frecuencia se presenta entre estar de acuerdo y totalmente de acuerdo, con solo unas pocas repeticiones por debajo, sin embargo, no sucede lo mismo cuando se trata de trabajar mapas conceptuales y redes semánticas, donde se muestra una dispersión en las cinco escalas de valoración. Por su parte, en relación con las estrategias de aprendizaje, se valoraron aspectos como la repetición de forma escrita o hablada para recordar conceptos importantes, elaboración de resúmenes o notas libres que relacionen la información 
con el fin de crear uniones entre lo nuevo y lo familiar, agrupación de información, planificación y manejo de tiempo. En este sentido, la gráfica muestra que los estudiantes tienen una tendencia a implementar estas estrategias para su aprendizaje.

Por otro lado, la figura 3 muestra los resultados asociados a la dimensión de materiales y recursos. En este sentido, la primera indagación tiene que ver con la utilización de libros, revistas o bases de datos institucionales, donde se evidencia una mayor tendencia en no estar de acuerdo ni en desacuerdo, sin embargo, contrario a estos, se puede afirmar que el material utilizado es producción propia del profesor, lo que explica la poca utilización de materiales externos.

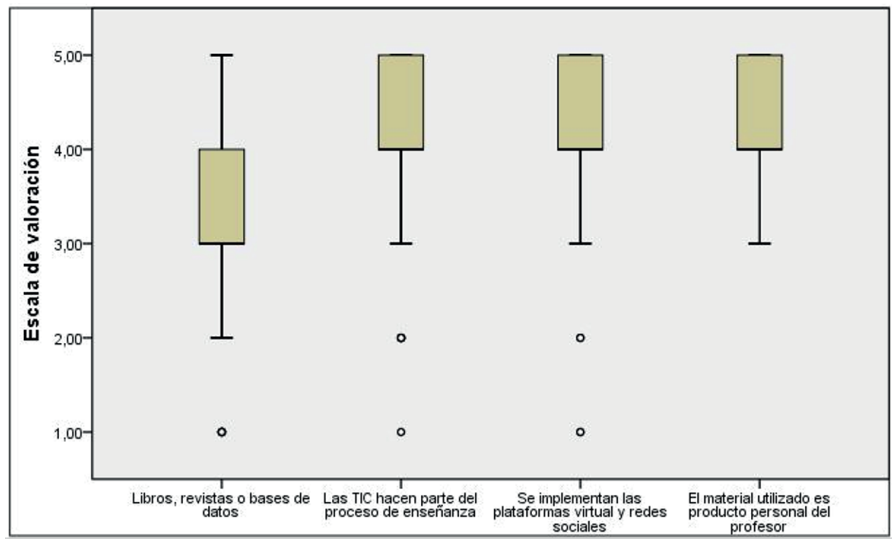

Figura 3. Percepción de los estudiantes frente a los materiales y recursos implementados

En cuanto a las Tecnología de Información y Comunicación (TIC), representadas especialmente por la plataforma virtual institucional (aula virtual) y las redes sociales, se evidencia claramente que se encuentran incorporadas en el proceso de enseñanza y aprendizaje, en este sentido se constituye una combinación y habilidades digitales que dan un enfoque al uso de la mente humana, la creatividad y la innovación (vivas, Cuberos, Castellanos y Gelvez, 2017). Por último, la figura 4 muestra los resultados asociados a la dimensión de evaluación, donde se tuvieron en cuenta aspectos como la finalidad o función, extensión global y parcial, agente evaluador, y enfoque grupal e individual.

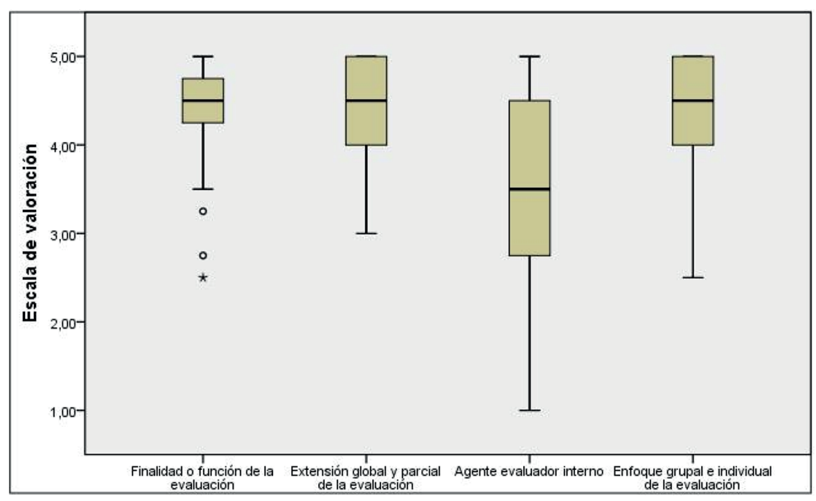

Figura 3. Percepción de los estudiantes frente a la evaluación
Con respecto a la finalidad o función de la evaluación, el instrumento indagaba asuntos como la implementación de al menos una prueba diagnóstica, actividades evaluativas constantes, pertinencia de la evaluación, y acciones de mejora de acuerdo a los resultados, donde se evidencia una fuerte tendencia de cumplimiento. Ahora bien, en cuanto a la extensión global y parcial, y el enfoque grupal e individual de la evaluación, se puede afirmar que se implementan constantes actividades evaluativas con un buen enfoque, ya sea personal o grupal, mientras que, en referencia al agente evaluador, se evidencia poca participación del estudiante. En este punto es importante resaltar que la evaluación requiere una revisión metacognitiva partiendo de una reflexión con la participación de todos los actores involucrados en el proceso educativo (Vivas, Cuberos y Gelvez, 2017).

Además de la información anterior, también se cuenta con el análisis realizado a los documentos institucionales y del profesor como programas analíticos, listas de asistencia, guías de trabajo y evaluaciones. Consecuentemente al contrastar los resultados de la encuesta con los encontrados en los programas analíticos y listas de asistencia, se encontró que las estrategias de enseñanza y aprendizaje implementadas en clase no se mencionan en los programas analíticos de las asignaturas.

Por otro lado, al observar el uso de la plataforma virtual, se encontró que los profesores efectivamente comparten material propio, sin embargo, este material no se encuentra diseñado bajo el modelo o formato que propone la institución para la elaboración de guías de trabajo en clase. Otro factor que se analizó en los programas analíticos es la evaluación que realiza los docentes, donde se puede observar que las más utilizadas son las de agente evaluador interno, sin embargo no se cuenta con un modelo específico de autoevaluación, heteroevaluación y coevaluación, adicional a esto, en los programas analíticos los profesores mencionan que las evaluaciones parciales se realizarán de forma objetiva en un $50 \%$, pero al revisar las evaluaciones aplicadas se puede observar contradicción pues se evalúa solo contenido y procedimientos.

De acuerdo con los resultados derivados de la aplicación del cuestionario y los documentos analizados, se puede concluir que la percepción que tienen los estudiantes frente a la praxis de los profesores en torno a la metodología, técnicas e instrumentos es muy buena, sin embargo se hace esencial socializar con los profesores las diferentes metodologías para el desarrollo de competencias transversales, ya que estas no son muy claras. Por último, se evidencia la necesidad de socializar la evaluación basada en competencias, al igual que definir modelos referentes a su modalidad, ya sea diagnóstica, formativa o sumativa, así como lo relacionado con el ente evaluador interno (autoevaluación, heteroevaluación y coevaluación). 


\section{Conclusiones}

Con relación a la percepción de los estudiantes frente a la praxis de los profesores, se puede concluir de forma general que es positiva, sin embargo se evidencia una falta de coherencia entre la practica en el aula y la planificación presentada en los documentos institucionales, lo que no permite generar propuestas de mejoramiento institucional asertivas, pues la base esencial para el diseño de estas estrategias son los documentos suministrados por el profesor.

Asimismo se destaca la implementación de materiales propios diseñados por el profesor, y el fuerte manejo de las TIC, en especial la plataforma virtual de la que dispone la institución, no obstante es importante encontrar un equilibrio que beneficie la incorporación de recursos bibliográficos disponibles en la institución, ya sean físicos o digitales.

El cuanto al proceso de evaluación, se concluye que existen evidencias claras sobre la tendencia a la valoración de contenidos y procedimientos por parte del profesor, dejando de lado aspectos importantes como la evaluación autentica del conocimiento, la coevaluación por pares, y la participación del estudiante como agente evaluador y protagonista de su propio proceso de aprendizaje.

\section{Referencias}

Balbo, S, J. (2008). Guía práctica para la investigación sin traumas II. (2a ed.). San Cristóbal: FEUNET.

Benhayón, M. (2006). Desarrollo de competencias a través del estudio de la matemática en estudiantes de ingeniería de la Universidad Metropolitana de Caracas. (Tesis doctoral). Universidad Metropolitana de Caracas, Caracas, Venezuela.

Díaz, B, F. y Hernández, R, G. (1999). Estrategias docentes para un aprendizaje significativo. México: McGraw Hill.

Ferrer, M, C. (1997). El diseño de la formación profesional. Revista interuniversitaria de formación del profesorado, 11(30), 69-76.

Gardner, H. (1987). Las estructuras de la mente. La teoría de las inteligencias múltiples. México: Fondo de Cultura Económica.

Gelvez, E. (2016). Competencias transversales en matemáticas que fortalecen la formación profesional del Ingeniero de Sistemas de la Universidad Simón Bolívar Sede Cúcuta (trabajo de grado de maestría). Universidad Nacional Experimental del Táchira, San Cristóbal, Venezuela.

Gelvez, E. y Vivas, M. (2017). Aspectos cognitivos, metodológicos y etimológicos que subyacen en la formación por competencias del estudiante de Ingeniería de Sistemas. En J. Gómez, A. J. Aguilar, S. S. Jaimes, C. Ramírez, J. P. Salazar, J. C. Contreras y J. F. Espinosa (Ed). Prácticas pedagógicas. (pp. 979-999). Maracaibo: Universidad del Zulia.
Hernández, S, R., Fernández, C, C. y Baptista, L, P. (2014). Metodología de la investigación. (6a ed.). Bogotá: McGraw-Hill.

Hymes, D. (1996). Acerca de la competencia comunicativa. Revista forma y función, (9), 13-37. Recuperado de: http://www.revistas.unal.edu.co/index.php/formayfuncion/article/view/17051/17909

Leal, G, A. (2014). Implicaciones pedagógicas del enfoque de formación por competencias en la configuración de la práctica pedagógica de los docentes de la Universidad Simón Bolívar extensión Cúcuta. (Trabajo de grado de maestría). Universidad Francisco de Paula Santander, Cúcuta, Colombia.

McClelland, D. C. (1973). Testing for competencies rather than intelligence. American Psychologist, 28, 1-14. Recuperado de: http://www.lichaoping.com/wp-content/ ap7301001.pdf

MINISTERIO DE EDUCACIÓN NACIONAL. (2008). Propuesta de lineamientos para la formación por competencias en educación superior. Artículo en línea. Recuperado de: http://www.mineducacion.gov.co/1621/ articles-261332_archivo_pdf_lineamientos.pdf

Navarro, C, L. (2009). Desarrollo, ejecución y presentación del proyecto de investigación. Caracas: Melvin.

Sternberg, R.J. (1997). Successful intelligence. New York: Simon and Shuster.

Tobón, S. (2006). Aspectos básicos de la formación basada en competencias. Talca: Proyecto Mesesup. Recuperado de: https://maristas.org.mx/gestion/web/doctos/aspectos_basicos_formacion_competencias.pdf

Tobón, S. (2008). La formación basada en competencias en la formación superior: El enfoque complejo. Bogotá: Instituto Cife.ws. Recuperado de: https://cmapspublic3. ihmc.us/rid=1LVT9TXFX-1VKC0TM-16YT/Formaci $\%$ C3\%B3n $\% 20$ basada $\% 20$ en $\% 20$ competencias $\% 20$ (Sergio\%20Tob\%C3\%B3n).pdf

Torrado, R, A. (2015). Diseño curricular para favorecer las competencias genéricas en el contexto del horizonte pedagógico sociocrítico. (Trabajo de grado de maestría). Universidad Simón Bolívar, Cúcuta, Colombia.

Trejos, O, I. (2011). Hacia una verdadera relación entre un programa de ingeniería y el concepto de formación integral. Revista avances: investigación en ingeniería, 13(6), 4562. Recuperado de: http://dialnet.unirioja.es/servlet/articulo? codigo $=3705102$

Urrego-Giraldo, G. y Giraldo, G, L. (2009). Incorporación de las competencias al modelo de construcción de currículos de ingeniería basados en problemas: el caso de Ingeniería de Sistemas. Revistas avances en sistemas e informática, 6(3), 33-44.

Vivas, M., Cuberos, M. A., Castellanos, M. y Gelvez, E. (2017). Una aproximación a la evaluación del desempeño basado en competencias en un entorno virtual. En M. E. Graterol, M. I. Mendoza, R. Graterol, J. C. Contreras y J. F. Espi- 
nosa (Ed). Las tecnologías de información y comunicación y la gestión empresarial. (p. 351-383). Maracaibo: Universidad del Zulia.

Vivas, M., Cuberos, M. A. y Gelvez, E. (2017). La evaluación por competencias centrada en el desempeño de los estudiantes y su correlación con la evaluación auténtica de los aprendizajes. En J. Gómez, A. J. Aguilar, S. S. Jaimes, C. Ramírez, J. P. Salazar, J. C. Contreras y J. F. Espinosa (Ed). Prácticas pedagógicas. (p. 120-150). Maracaibo: Universidad del Zulia. 